Aus der Abteilung für Haut- und Geschlechtskranke der k. k. Krankenanstalt Rudolphstiftung (Primararzt Dr. v. Zumbusch) in Wien.

\title{
Ein Fall von Naevus Pringle und Neurofibromatosis [v. Recklinghausen]. \\ Von
}

\author{
Dr. A. Hintz, \\ Assistent.
}

(Hiezu Taf. XI.)

Im Jahre 1890 hat Pringle Fälle von sogenanntem Adenoma sebaceum beschrieben; er hat als solches eine Hautaffektion, deren histologischer Bau schwer zu deuten schien, bezeichnet. In der Folge wurde eine Reihe analoger Fälle von verschiedenen Autoren publiziert, doch wurden dieselben nicht alle gleichartig aufgefaßt.

Jadassobn hat sich zu dieser Frage in seiner Arbeit: Bemerkungen zur Histologie der systematisierten Naevi und über "Talgdrüsen Naevi" geäußert und sich dahin ausgesprochen, daß derartige Affektionen, wie sie auch von $\mathrm{Bal}$ zer, Ménétrier, Barlow u. a. beschrieben wurden, weder als Adenome noch als Hyperplasien der Talgdrüsen aufzufassen sind, sondern lediglich ${ }_{n}$ auf einer abnormen Keimesanlage beruhen, für die wir einen path. anat. Namen nicht besitzen, höchstens noch den Begriff Naevus". Außer zahlreichen Anderen sind in neuerer Zeit auch von Reitmannn (diese Zeitschr. Bd. LXXXIII) hierher gehörige Fälle kritisch besprochen worden. Auch er kommt (in Übereinstimmung mit 
Riebl) zu dem Ergebuis, daß die Affektion als Naevus in weiterem Sinne aufzufassen sei.

Die Krankengeschichte unseres Falles ist folgende:

Im Jänner 1910 wurde eine 27jährige Frau N. N. auf die Abteilung aufgenommen, welche wegen der frisch akquirierten Lues (Sklerosis labii major. sinistri und Exanthema maculosum) zur Behandlung von ihrem Kassenarzte geschickt wurde. Neben der Syphilis fiel die Veränderung auf, welche vor allem ihr Gesicht zeigte (s. Tafel XI). Das ganze Gesicht ist blaurot und bei näherer Betrachtung findet man eine Aussaat von kleinen bis stecknadelkopfgroßen lividen Knötchen, die vornehmlich an der Nasolabialfalte sitzen und hier Linsengröße erreichen, doch auch über Oberlippe, Kinn und Wange sichtbar sind, in Haufen liegen, und nirgend Konfluenz zeigen. Die Knötchen sind schon makroskopisch erkennbar stark vaskularisiert, wodurch eben die blaue Farbe des Gesichtes bedingt ist. Beim Tasten fühlen sie sich sehr derb an, sind verschieblich und völlig indolent, beim Ausquetschen oder Anstechen leicht blutend. Die beiden Gesichtshälften sind ziemlich symmetrisch von diesen Knōtchen bedeckt, am dichtesten sitzen sie an den Nasenflügeln traubenartig gruppiert, wo sie auch die bedeutende Größe erreichen. An der linken Stirnhälfte u. zw. von der Haargrenze gegen die Mitte der l. Augenbraue zustrebeud ein seichter derber, breit aufsitzender, ca. $2 \frac{1}{2} \mathrm{~cm}$ langer blaßgelber Tumor, der sich histologisch als Keloid erweist. Der Hals der Patientin erscheint durch die nicht unbeträchtlich große Struma dick und zu beiden Seiten an der Stelle, wo der Hemdrand zu liegen plegt, finden sich strichförmig angeordnet horizontal nach hinten ziehend wieder bis linsengroße, gelbbraun pigmentierte weiche gestielte Tumoren in ziemlicher Zahl, die als Fibromata mollusca imponieren.

Der Rücken der Patientin ist leicht kyphoskoliotisch verkrümmt, die Kückenhaut blaß3 und einzelne milchkaffeebraune Pigmentflecke sind da und dort zerstreut aufzufinden.

Die Fingernägel und Zähennägel weisen an der Matrix einzelne hornartige spitze kleinste Papillome auf, die ganz schmerzlos sind.

Die Anamnese bei der Frau zu erheben gestaltete sich etwas scbwierig, weil ihre intellektuellen Fähigkeiten entschieden unter der Norm sind. $\mathrm{Zu}$ eruieren war, daß die Veränderungen, welche die Haut zeigt, in frühester Kindheit bemerkt wurden, dals sie niemals Schmerzen in der Gesichtshant empfand und $\mathrm{dab}$ sie überhaupt nie krank gewesen sei. Die Verkrümmung der Wirbelsäule war ihr bewußt, doch hinderte sie sie nicht bei ihrer Arbeit. Über ähnliche Fälle in ihrer Familie weiß sie nichts.

Die Patientin ist jetzt im 7. Monat gravid und gibt an, dab weder zur Zeit ihrer Schwangerschaft, noch vor der Zeit die Knötchen im Gesicht gewachsen oder zurückgegangen seien. Die Menses begannen bei ihr im 14 oder 15. Lebensjahre, waren regelmäßig und mit dem Einsetzen der ersten 


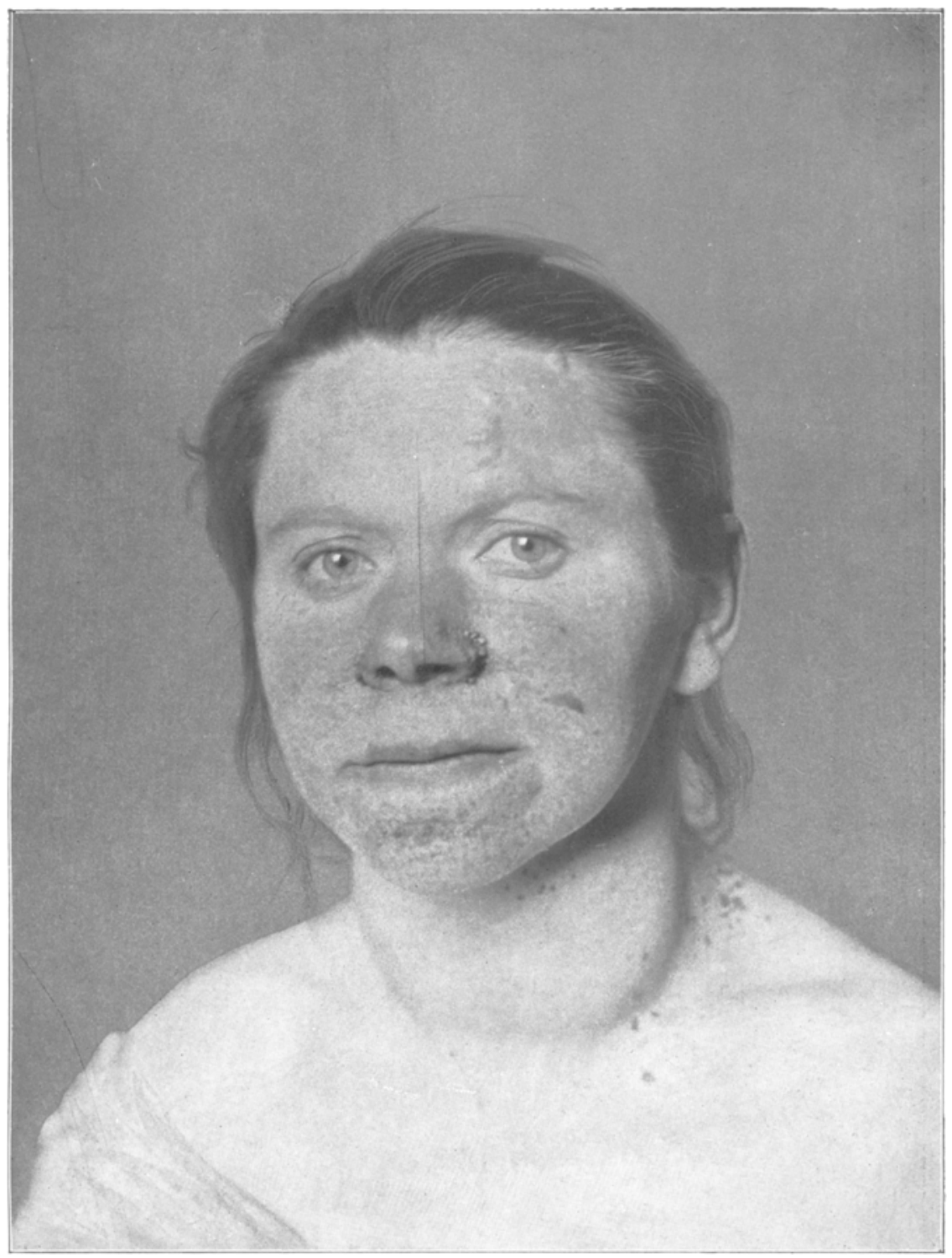

II intz: Fin Fall von Nacvus Pringle und Neurofibromatosis. 
Menstruation sind die Tumoren im Gesicht nicht gewachsen. Allerdings sind alle diese Angaben mit Reserve aufzunehmen, da die Patientin sehr imbezill ist. Schreiben und Lesen hat Patientin angeblich gelernt, scheinbar aber nicht erlernt.

Was den Status praesenz betrifft kann noch zugefügt werden, daß die Frau einen kräftigen Knochenbau zeigt, sie ist von mittlerer Gröle. Der Gesichtsausdruck stupid, der Hals durch eine ca. faustgroße Struma entsprechend dick kolbig. Das Abdomen der 7 monatlichen Gravidität entsprechend stark vorgewölbt.

Zur mikroskopischen Untersuchung wurden der Patientin zu beiden Seiten an den Nasenflügeln bis über die Nasolabialfalte, also diejenigen Partien, welche die Veränderungen am deutlichsten zeigten, am Kinn wie den der Stirne, ziemlich große Stückchen exzidiert. Bei der Operation, die teilweise mit Schleichscher Anästhesie gemacht wurde, fiel die Hyp. ästhesie der Patientin auf, es konnten nämlich einzelne Hautstückchen gänzlich ohne Lokalanästhesie exzidiert werden, ohne daß Patientin Schmerzen äußerte.

Der pathologisch anatomische Befund der teils in Alkohol, teils in Formalin gehärteten Exzisionsstücke ist wie folgt:

Exzisionsstück rom linken Nasenflügel und Nasolabialfalte.

Bei schwacher Vergrößerung fallen vor allem die Exkreszenzen auf, durch welche die Hautoberfläche uneben und hökkerig ist. Über diesen Exkreszenzen findet sich eine hochgradige Verdünnung der Epithelschichte und nur an Basis und an den Seiten eine Andeutung vom Papillarkörper, an der Kuppe ist dieser nicht mehr zu finden. Unter der Basis der kleinen Tumoren finden sich vereinzelte Haarbälge ebenso an den Seitenteilen, sie münden nicht an der Kuppe, sondern weichen seitlich aus. Die Epithelzellen über den Kuppen sind schuppenförmig abgehoben und eleidinhältig. Unter dem Epithel der Kuppen und nur hier zahlreiches gelbes Pigment führende Chromatophoren. Fibrilläres Bindegewebe ist in den Exkreszenzen reichlich vorhanden, gegen die Mitte hin ist es ödematös und neben den Haarbälgen sind sehr weite Gefäße vorhanden, die sehr häufig nur aus einem Endothelrohr bestehen und kaum eine Adventitia angedeutet besitzen. Oft liegen diese Gefäße in reichlicher Menge und dicht nebeneinander. Um die Gefäße 
finden sich Infiltrate in spärlicher Menge, diese weisen Mastzellen und Lymphozyten auf. Die Färbung auf elastisches Gewebe zeigte ungemein schön, daß dieses Gewebe, welches sonst im Korium reichlich zu finden ist, hier fast gänzlich fehlt. Die elastischen Fasern hören an der Basis der Exkreszenzen nahezu vollständig auf und sind an den Kuppen sehr spärlich nur in zartesten Fäserchen nachweisbar. Die oben erwähnten erweiterten Gefäße besitzen keine Elastika.

Den eben geschilderten Befund ergeben alle Exzisionsstücke, ob sie nun vom Kinn oder von der Nasolabialgegend stammen oder der Wange entnommen sind. Erwähnenswert wäre noch das Vorhandensein von Talgdrüsen, wobei nicht unbemerkt bleiben soll, daß diese wohl reichlich, doch völlig der Norm entsprechend zu finden sind, also keine auffallende Vermehrung aufweisen und keine pathologischen Veränderungen erkennen lassen.

Die rom Halse entnommenen Hautstückchen geben den Befund der typischen Fibrome, von etwas ödematöser Strucktur und gerade die größeren Fibrome lassen deutlich sehr reichliche, aus Lymphozyten, Plasmazellen und spärlichen Mastzellen bestehende perivaskuläre Infiltrate erkennen. Die Gefäße sind auch hier sehr zartwandig und enorm weit. Desgleichen hört hier die Elastika in den größeren Knötchen an der Basis vollständig auf. Verfolgt man den Papillarkörper neben den Exkreszenzen so findet sich eine große Mannigfaltigkeit der Papillen in bezug auf ihre Größe und Gestalt. Es lassen sich alle Übergänge konstatieren; zwischen normalen Papillen bis zu bedeutend verbreiterten plumpen, denen uur geringe Erhebungen über die Oberfläche der Haut entsprechen und endlich den großen Fibromen.

Aus diesem Befunde geht hervor, daß die Tumoren den von Pringle beschriebenen Fällen analog sind, was ohne weiteres aus den Abbildungen Pringles ersichtlich ist.

Pringle bezeichnet seine Fälle als Adenomata sebacea.

In dem vorliegendem Fall waren zwar große Talgdrüsen nachweisbar, es scheint aber dieser Befund nicht das wesentliche der Affektion zu sein. Es war an keiner Stelle die Über- 
zeugung zu gewinnen, daß die Talgdrüsen größere Dimensionen besässen hätten, als sie sich de norma in dieser Gegend finden.

Die Bezeichnung Naevus ist also wohl mit Vorbehalt anzuwenden, da in den in Rede stehenden Gebilden der Nachweis von Naevuszellen nicht zu erheben ist.

Anatomisch resp. histologisch sind die Hautexkreszenzen fibromatöse Bildungen, deren gewebliche Selbständigkeit besonders darin zum Ausdruck kommt, dab ihnen die sonst in der Subkutis reichlich vorhandenen elastischen Fasern nahezu vollständig fehlen. Die in den Präparaten sichtbaren entzündlichen Infiltrate haben offenbar keinen ätiologischen Zusammenhang mit den Knötchen der Haut, sie sind als Effekte chronisch und auch mechanischer Reizungen anzusehen.

Faßt man diese multiplen Knötchen als Fibrome auf, so würde sich aus dieser Anschauung unter Berücksichtigung anderer bei der Patientin vorhandenen Veränderungen eine, wie ich glaube, nicht uninteressante Beziehung zu einer andern auch mit Fibromatosis einhergehenden Erkrankung ergeben: dem Morbus Recklingha us e n. Iusbesondere kämen in dieser Hinsicht in Betracht die am Körper zerstreuten zahlreichen Pigmentflecke und Knötchen, die reichlichen Fibrome der Halsgegend, die Kyphoskolisis und die Imbezillität der Patientin, durchaus Merkmale für den Symptomenkomplex, wie sie dem Morbus Recklinghausen zukommen, wenn letzteres auch ein häufiges Vorkommen bei den Fällen von Naevus Pringle ist.

Fassen wir den ganzen Symptomenkomplex zusammen, so ergibt sich, daß wir bei der Patientin beide Krankheitsbilder, das des Naevus Pringle und das der von Recklinghau s e n schen Neurofiberomatose vereint finden. Wir sehen einerseits die charakteristischen roten Geschwïlstchen im Gesicht, die Papillome an den Endphalangen der Finger und Zehen, andrerseits weiche Fibrome, teils gestielt, teils breit aufsitzend, um die Schultern, am Hals, am Stamm, Pigmentflecke und die Verkrümmung der Wirbelsäule. Alles besteht seit Kindheit bei einem schwachsinnigen Individuum. Es liegt die Annahme nahe, daß beides als Anlagefehler zusammengehörig sei, was uns 
wohl zwingt die Bezeichnung Adenom aufzugeben und den Symptomenkomplex mit $\mathbf{J}$ adass ohn, Riehl und Reitmann als Naevus, wenn auch in einem weiteren Sinne, aufzufassen.

Die Erklärung der Abbildungen auf Taf. XI. ist dem Texte zu entnehmen. 